\title{
Complete Genomic Sequence of Goose-Origin Reovirus from China
}

\author{
Tao Yun, Weicheng Ye, Zheng Ni, Liu Chen, Bin Yu, Jionggang Hua, Yan Zhang, and Cun Zhang \\ Institute of Animal Husbandry and Veterinary Medicine, Zhejiang Academy of Agricultural Sciences, Hangzhou, China
}

\begin{abstract}
We report the full-genome sequence of a goose-origin reovirus (GRV) strain 03G from Zhejiang Province, China. This is the first report of the complete genomic sequence (segments 1 to 10) of GRV. Phylogenetic analyses of the sequence suggest that GRV $03 \mathrm{G}$ represents a new species distinct from other established species within the avian reovirus (ARV) group of orthoreoviruses.
\end{abstract}

$A^{v}$ vian reoviruses (ARVs) are members of the genus Orthoreovirus in the family Reoviridae and were first isolated from chickens in 1954 (4). ARV is the major etiological agent of viral arthritis and tenosynovitis of chickens (9). The virus also infects a wide variety of other avian species worldwide, including turkeys, Muscovy ducks, geese, and several wild bird species (5). As in all reoviruses, the ARV virion is nonenveloped and composed of a double concentric icosahedral capsid. The genomes of ARVs consist of 10 segments of double-stranded RNA which are divided into three size classes: large (L1 to L3), medium (M1 to M3), and small ( $\mathrm{S} 1$ to $\mathrm{S} 4)$. These segments encode at least eight structural proteins $(\lambda \mathrm{A}, \lambda \mathrm{B}, \lambda \mathrm{C}, \mu \mathrm{A}, \mu \mathrm{B}, \mu \mathrm{BC}, \mu \mathrm{BN}, \sigma \mathrm{C}, \sigma \mathrm{A}$, and $\sigma \mathrm{B})$ and four nonstructural proteins ( $\mu$ NS, P10, P17, and $\sigma \mathrm{NS})(2,3,7,8)$. Several differences between chicken-origin ARV and duck-origin reovirus (DRV) have been noted, including different antigenicities, host ranges, pathogenic properties, double-stranded-RNA (dsRNA) and protein profiles, and genomic coding assignments (10). To date, only six full genomic sequences of chicken-origin ARV strains have been reported; the complete sequences of gooseorigin reovirus (GRV) and DRV are lacking.

In the present study, we report the full genomic sequence (including noncoding regions) of GRV strain $03 \mathrm{G}$, which was isolated from the livers of 5 sick geese from Zhejiang Province, China, in 2003 that exhibited obvious clinical signs and lesions of hemorrhagic-necrotic hepatitis. Viral dsRNA from the GRV 03G-infected chicken embryo fibroblast cell line (DF-1) was isolated using RNAiso Plus (TaKaRa), and full-length cDNA copies of each genomic segment were reverse transcribed and amplified in a sequence-independent manner using the anchor spacer-ligation method as described previously $(1,6)$. These PCR products were purified and sequenced on an ABI 3730 capillary sequencer (Applied Biosystems), using phased primers to generate near-terminal sequences and segment-specific walking primers to determine the full nucleotide sequence. The sequences were analyzed with Vector NTI Advance 9 software, and phylogenetic trees were constructed with Mega 5.0 software.

The complete genome of GRV 03G is $24,218 \mathrm{bp}$, and the sizes of segments 1 through 10 are as follows: L1, 3,958 bp; L2, 3,829 bp; L3, 3,907 bp; M1, 2,283 bp; M2, 2,158 bp; M3, 1,996 bp; S1, 1,568 bp; S2, 1,326 bp; S3, 1,202 bp; and S4, 1,191bp. The deduced lengths of eight structural proteins and three nonstructural proteins are as follows: $\lambda \mathrm{A}, 1,293$ amino acids (aa); $\lambda \mathrm{B}, 1,259 \mathrm{aa} ; \lambda \mathrm{C}$, $1285 \mathrm{aa} ; \mu \mathrm{A}, 732 \mathrm{aa} ; \mu \mathrm{B}, 676$ aa; $\sigma \mathrm{A}, 416 \mathrm{aa}$; and $\sigma \mathrm{B}, 367$ aa; and $\sigma \mathrm{C}, 321 \mathrm{aa}$. The sequence of $\sigma \mathrm{C}$ of GRV 03G, coded by the S1 segment, was compared with its counterpart in the other ARV strain. Phylogenetic analysis of this gene indicates that GRV 03G belongs to neither the chicken reovirus cluster nor the duck reovirus cluster. We suggest that GRV 03G be recognized as a new species within the ARV group.

Nucleotide sequence accession numbers. The full genomic sequence of GRV strain 03G was deposited in GenBank. The accession numbers JX145328 to JX145337 correspond to the virus RNA segments 1 through 10 , respectively.

\section{ACKNOWLEDGMENTS}

We are thankful to Joel D. Baines and Kui Yang (Department of Microbiology \& Immunology, Cornell University) for critical review and suggestions for the manuscript.

This work was supported by grants from Modern Agro-industry Technology Research System (nycytx-45-11), the Scientific and Technological Innovation Team of Zhejiang Province, and the Technological Innovation Capability Project of Zhejiang Academy of Agricultural Sciences (2010R22Y01D01).

\section{REFERENCES}

1. Attoui H, et al. 2000. Strategies for the sequence determination of viral dsRNA genomes. J. Virol. Methods 89:147-158.

2. Benavente J, Martínez-Costas J. 2007. Avian reovirus: structure and biology. Virus Res. 123:105-119.

3. Bodelón G, Labrada L, Martínez-Costas J, Benavente J. 2001. The avian reovirus genome segment $\mathrm{S} 1$ is a functionally tricistronic gene that expresses one structural and two nonstructural proteins in infected cells. Virology 290:181-191.

4. Fahey JE, Crawley JF. 1954. Studies on chronic respiratory disease of chickens II. Isolation of a virus. Can. J. Comp. Med. Vet. Sci. 18:13-21.

5. Liu QF, et al. 2011. Isolation and characterization of a reovirus causing spleen necrosis in Pekin ducklings. Vet. Microbiol. 148:200-206.

6. Maan S, et al. 2007. Rapid cDNA synthesis and sequencing techniques for the genetic study of bluetongue and other dsRNA viruses. J. Virol. Methods 143:132-139.

7. Shmulevitz M, et al. 2002. Sequential partially overlapping gene arrangement in the tricistronic S1 genome segments of avian reovirus and Nelson Bay reovirus: implications for translation initiation. J. Virol. 76:609-618.

8. Varela R, Benavente J. 1994. Protein coding assignment of avian reovirus strain S11343. J. Virol. 68:2974-2981.

9. Wilcox GE, Robertson MD, Lines AD. 1985. Adaptation and characteristics of replication of a strain of avian reovirus in Vero cells. Avian Pathol. 14:321-328.

10. Zhang Y, et al. 2007. Characterization of M-class genome segments of muscovy duck reovirus S14. Virus Res. 125:42-53.

Received 29 June 2012 Accepted 3 July 2012 Address correspondence to Cun Zhang, cunzh2004@yahoo.com.cn. Copyright ( 2012, American Society for Microbiology. All Rights Reserved. doi:10.1128/JVI.01692-12 